\title{
Ability of Granger Causality Analysis to Detect Indirect Links: A Simulation Study
}

\author{
N. W. Falasca \\ $B I N D$ - Behavioral Imaging and Neural Dynamics Center, \\ G. d'Annunzio University of Chieti-Pescara, ITALY \\ R. Franciotti* \\ Department of Neuroscience, Imaging and Clinical Science, \\ G. d'Annunzio University of Chieti-Pescara, ITALY
}

(Received 30 November, 2019)

\begin{abstract}
Granger causality (G-causality) has emerged as a useful tool to investigate the influence that one system can exert over another system, but challenges remain when applying it to biological data. Specifically, it is not clear if G-causality can distinguish between direct and indirect influences. In this study time domain G-causality connectivity analysis was performed on simulated electroencephalographic cerebral signals. Conditional multivariate autoregressive model was applied to 19 virtual time series (nodes) to identify the effects of direct and indirect links while varying one of the following variables: the length of the time series, the lags between interacting nodes, the connection strength of the links, and the noise. Simulated data revealed that weak indirect influences are not identified by Gcausality analysis when applied on covariance stationary, non-correlated electrophysiological time series.
\end{abstract}

PACS numbers: $87.10 .+\mathrm{e}, 07.05 . \mathrm{Kf}$

Keywords: Granger causality, simulation, temporal window, lags, gain, noise

DOI: https://doi.org/10.33581/1561-4085-2020-23-2-121-124

\section{Introduction}

The characterization of the functional connectivity (FC) patterns among brain areas is a key challenge in neuroscience. Recent advances have shown that FC patterns of human brain networks can be non-invasively characterized by electroencephalography (EEG) recordings. Among the statistically principled techniques which estimate the FC and the direction of influence in EEG time series, Granger causality (G-causality) has emerged as a useful and the most prominent tool [1, 2]. G-causality calculates the directed connectivity between two time series based on the notion that information in the past of the one helps to predict the future of the other with greater accuracy than by only considering the past of itself [3]. However, challenges remain on the ability of G-causality to infer the

\footnotetext{
${ }^{*}$ E-mail: raffaella.franciotti@itab.unich.it
}

influence among nodes of a biological network, to distinguish between direct and indirect influences and to its relation with the strength of the neural interactions. The aim of this study is to evaluate the reliability of G-causality results on indirect influences and to estimate how the results are modulated by the strength of the link between nodes.

\section{Methods}

\subsection{Simulated data}

G-causality analysis can be performed under these assumptions:

- time series are not correlated;

- the time series are stationary, more specifically they must be covariance stationary (also known as weak or widesense stationarity, i.e., the means and 
variances of the time series are stable over time);

- the model does not have any unit roots which are one cause for non-stationarity.

In this study simulated time series $\mathrm{X}(\mathrm{t})$ were generated in order to guarantee these assumptions, summing the sinusoidal signals, with a random phase for each frequency $i$ and according to the following formula:

$$
X(t)=\sum_{i=1}^{n} A_{i} \sin \left(2 \pi \cdot f_{i} \cdot t+\varphi_{i}\right)
$$

where $n=2460$ (for a sampling rate of $256 \mathrm{~Hz}$ and the frequency resolution of $0.05 \mathrm{~Hz}$ ),$A_{i}$ and $f_{i}$ are amplitude and frequency,

$$
\varphi_{i}=\operatorname{randn} \cdot 2 \pi
$$

randn is a random scalar function drawn from the standard normal distribution. G-causality is not based on the phase differences between time series, thus the results were not influenced by the distortion of the time series by the randomization of the phases.

In addition, randomly generated white Gaussian noise was produced by means of the awgn(snr) function from matlab library and was super-imposed to each time series, obtaining a simulated raw time series for each of the 19 EEG channels (nodes). The scalar parameter snr specifies the signal-to-noise ratio per sample, in $\mathrm{dB}$.

The link from the time series $Y(t)$ to the time series $X(t)$ was simulated according to the following formula:

$$
X(t+l a g)=X(t)+A m p \cdot Y(t),
$$

where

$$
t=1, \ldots,(\text { length }(X)-l a g)
$$

The lag and it Amp parameters were the delay and the connection strength factor in the influence from the time series $\mathrm{Y}$ (driver) to the time series X (recipient).

Two examples of simulated data were performed. The first example consisted of 19 time series (nodes), with five sources (drivers), seven sinks (recipients) and seven links. The lags between causal interacting nodes and the gain values (i.e. the connection strength factor which multiplied the amplitude of the driver signal) between nodes are reported for the starting designed structure in 1 . We evaluated the influence of the length of the time series on Gcausality results, changing the time points from $512(2 \mathrm{~s})$ to $12800(50 \mathrm{~s})$ with a step of 256 . Then, for a selected length of the time series, we changed the lags adding a value ranging from -9 to 30 time points with step 1 to the lag values. Afterwards we added value ranging from -0.2 to 0.2 with step 0.01 to the connection strength factor. To simulate the presence of an indirect influence we added a latent $20^{\text {th }}$ time series to simulate a link from node 10 to node 20 and from node 20 to node 13. The aim was to verify if G-causality would pick the false influence from 10 to 13 .

A second example of simulation was performed consisting of 19 nodes with 12 sources, 11 sinks and 14 links. The indirect influence from node 10 to node 13 was also included with the same characteristics as in the first example. Figure 1 shows the designed structures for the two examples.

\subsection{G-causality analysis on simulated} data

Time domain G-causality connectivity analysis was applied to identify patterns of causal influence between nodes. According to linear vector autoregressive (VAR) models, two wide-sense stationary time series $X(t)$ and $Y(t)$ can be explained by their own past by means of a linear model with coefficients $a_{j}$ and $b_{j}$ and prediction errors $\epsilon_{1}(t)$ and $\eta_{1}(t)$ respectively:

$$
\begin{aligned}
& X(t)=\sum_{j=1}^{m} a_{j} X(t-j)+\epsilon_{1}(t) \\
& Y(t)=\sum_{j=1}^{m} b_{j} Y(t-j)+\eta_{1}(t)
\end{aligned}
$$

According to Granger [3] the temporal dynamics of $X(t)$ and $Y(t)$ (both of length $T$ ) can be described also including in the model the information on the past of the other time series, with prediction errors $\epsilon_{2}(t)$ and $\eta_{2}(t)$ 


$$
\begin{gathered}
X(t)=\sum_{j=1}^{m} a_{j} X(t-j)+\sum_{j=1}^{m} b_{j} Y(t-j)+\epsilon_{2}(t) \\
Y(t)=\sum_{j=1}^{m} c_{j} Y(t-j)+\sum_{j=1}^{m} d_{j} X(t-j)+\eta_{2}(t),
\end{gathered}
$$

where $m$ is the maximum number of lagged observations (the model order, $m \ll T$ ), whereas $b_{j}$ and $d_{j}$ are the gain factors of $Y(t)$ (driver) influencing $X(t)$ (recipient), and of $X(t)$ (driver) influencing $Y(t)$ (recipient). The linear influence from $X(t)$ to $Y(t)\left(F_{X \rightarrow Y}\right)$ and from $Y(t)$ to $X(t)$ $\left(F_{Y \rightarrow X}\right)$ can be calculated as the log ratio between the variances of the residual errors:

$$
\begin{aligned}
& F_{X \rightarrow Y}=\log \left(\frac{\operatorname{var}\left(\eta_{1}\right)}{\operatorname{var}\left(\eta_{2}\right)}\right) \\
& F_{Y \rightarrow X}=\log \left(\frac{\operatorname{var}\left(\epsilon_{1}\right)}{\operatorname{var}\left(\epsilon_{2}\right)}\right)
\end{aligned}
$$

G-causality analysis is generalized to the multivariate (conditional) case in which the Gcausality of $Y(t)$ on $X(t)$ is tested in the context of multiple additional variables when all other variables are also included in the regression model. In our simulation study, conditional multivariate VAR (MVAR) model was applied to 19 time series to estimate G-causality connectivity [4] using the in-house software BSMART, a MATLAB/C Toolbox implemented to analyse brain [5]. The method of ordinaryleast-squares was used to compute the regression coefficients. The F-statistic, Bonferroni-corrected (nominal p value of 0.05 , then divided for multiple comparisons by $n \cdot(n-1)$ (where $n=19)$, was applied to the coefficients of the MVAR model. The Akaike information criterion [6] was used to estimate the order of the model [7]. Covariance stationarity was checked by using the DurbinWatson test, based on MATLAB code [4] and the Dickey-Fuller test $(p<0.01)$ to identify unit roots. The consistency of the MVAR model, which ensures that it properly represents the data, was verified by the tests proposed by [8], whereas the Durbin-Watson statistics assessed whether the residuals are uncorrelated.

\section{Results}

For the first and the second example the false indirect influence from node 10 to 13 was revealed for the length of the time series longer than 4608 time points. For the first example the indirect influence was revealed when the adding values of the lags to the designed structure ranged from -9 (lags between time series from 1 to 7 time points) to 0 (lags between time series from 10 to 16 time points), for the second example when the adding values of the lags ranged from -9 to -1 (lags between time series from 9 to 15 time points). For the first example the indirect influence was revealed when the connection strength factor was higher than 0.33 (the adding value to the connection strength factor was 0.03), for the second example it was instead revealed when the connection strength from node 20 to 13 was higher or equal to 0.5 (adding value of 0.05 ). The false indirect influence was never revealed by Gcausality analysis, increasing the noise, for the first as well as the second example.

\section{Conclusions}

In this study we generated 19 virtual simulated covariance stationary time series with a designed structure of influence among nodes, which we referred to as a ground truth structure. Conditional G-causality analysis in the time domain was performed to test its reliability on indirect links while varying one of the following variables: the number of time points used to perform G-causality, the lags between interacting nodes, the connection strength of the links, and the noise. The evaluation of the G-causality reliability on the indirect link showed that Gcausality picked the false link as true when the length of the temporal window was higher than 4608, time points, the lags of the designed structure was lower than 15 time points and the connection strength factor was higher than 0.3 time the amplitude of the driver signal for the first example and 0.5 for the designed structure with higher number of links (second example). To note that in both examples, the connection factor between node 10 to the latent node 20 was fixed to 0.5 , whereas the connection factor between the latent node 20 to node 13 varied. Additional simulations should be performed varying also the connection strength factor between node 10 to 
node 20 .

A

First example

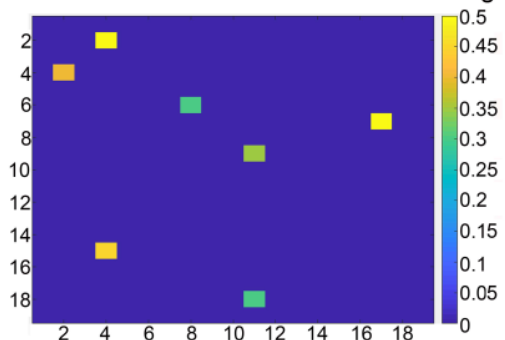

Second example

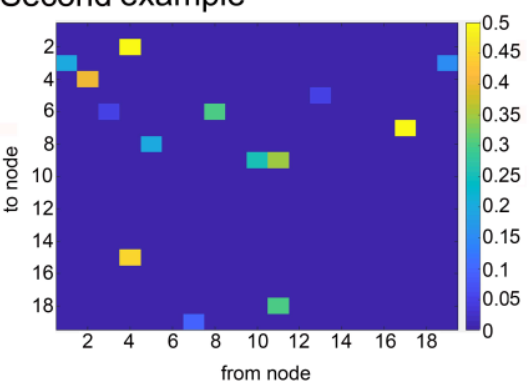

B
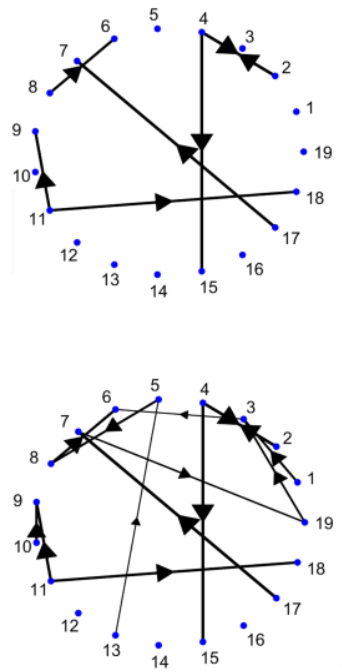

$\mathrm{C}$

STARTING VALUE OF THE DESIGNED STRUCTURE

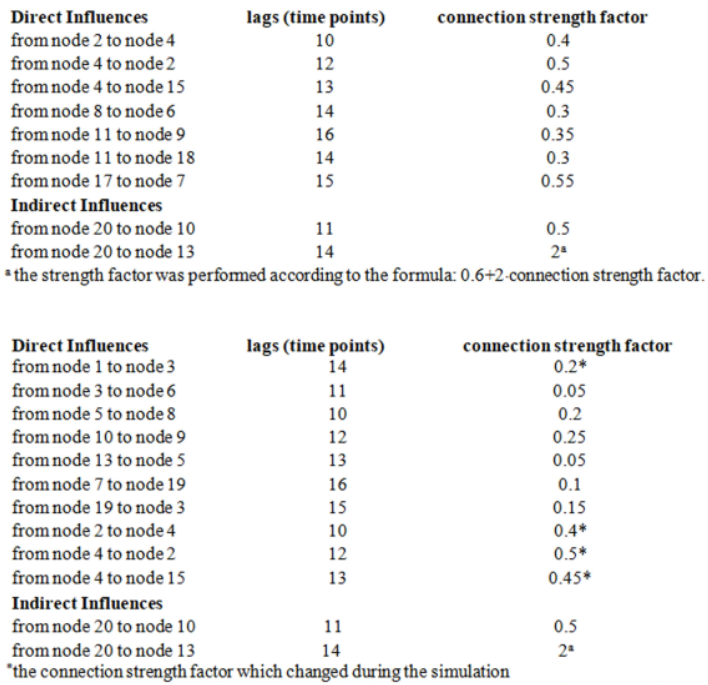

FIG. 1. (color online) Designed structures for the two examples. The links are shown by the G-causality matrices with their connection strength factors (as indicated by the colorbars for the first and the second examples (A), and by arrows indicating the direction and versus of the links between nodes $(\mathbf{B})$. Starting values of the lags and of the factors which multiply the amplitude of the driver signal of the link for the first and the second example (C).

\section{References}

[1] A. Brovelli, M. Z. Ding, A. Ledberg, Y. H. Chen, R. Nakamura, and S. L. Bressler, Proc. Natl. Acad. Sci. U.S.A. 101(26), 9849 (2004).

[2] A. K. Seth, A. B. Barrett, and L. Barnett, J. Neurosci. 35(8), 3293 (2015).

[3] C. W. Granger, Econ. J. Econ. Soc. 37(3), 424 (1969).

[4] A. K. Seth, J. Neurosci. Methods 186(2), 262 (2010).
[5] J. Cui, L. Xu, S. L. Bressler, M. Ding, and H. Liang, Neural Networks 186(2), 262 (2010).

[6] H. Akaike, IEEE T. Automat. Contr. 19(6), 71 (1974).

[7] S. L. Bressler and A. K. Seth, NeuroImage 58(2), 323 (1995).

[8] M. Ding, S. Bressler, W. Yang, and H. Liang., Biol. Cybern. 83(1), 35 (2000). 